\title{
Strategic implications for publishers*
}

\author{
Chair:
}

Rick Bowes, Society for Scholarly Publishing

Bowes continues a discussion begun in late 1998 at SSP's Top Management Round Table on Forces likely to Transform Scholarly Publishing.

Speakers:

Dennis Auld Senior Consultant, PSYCHInfo

Bonnie Lawlor President, Chescot Publishing

Linda Sacks Senior VP Marketing and Sales, BIOSIS

\section{The Way Forward for Secondary Publishers}

\section{The primary publisher platform}

A recent Society for Scholarly Publishing (SSP) Top Management Roundtable discussion on "Strategic Implications for Scholarly Publishing" revealed five key issues expected to impact the planning landscape in the next three to fives years. Rick Bowes, SSP's President, expands on these issues/themes:

- Theme \#1: The technology creates new dissemination options and many fragmented approaches. This fragmentation threatens to make the cooperative enterprise incoherent and unsustainable. Nonetheless, scholarly publishing will continue to meet the needs of society because scholars require the literature.

- Theme \#2: There is a need to reaffirm the value of the publishing process. There is more to publishing than rapid delivery. For example, publishers are experts at quality filtering, editing, production, marketing, distribution, subscription management, and other back-office activity. Grass-roots efforts are needed to educate end users, librarians, and researchers about these value-adds.

- Theme \#3: New structures and designs for information do require new formats, processes, and content (links). Archiving is going to be a critical issue, and there needs to be international, interdisciplinary standards to facilitate broad dissemination and archiving. Better interaction with IT/MIS personnel is also required.

- Theme \#4: Rights management and content management are becoming more important and more challenging. Publishers would like unlimited electronic rights without borders. But how do publishers deal with legacy content where rights weren't obtained? How should publishers handle corrections and forward linking? Content stewardship is expensive and time-consuming. Who should be the primary steward?

\footnotetext{
*In cooperation with the Society for Scholarly Publishing.
} 
- Theme \#5: Partnerships and alliances of many types will be essential, with the overall goal of improving products, increasing information's reach, and generating more revenues. Partnerships will sustain the more costlier publishing processes to come.

\section{Power to the secondaries!}

"Let me tell you why I am bullish about the future of secondary publishing", says Dennie Auld, Senior Consultant for PsycINFO. Secondaries possess and historic expertise in indexing, aggregation, comprehensive coverage, maintaining historical files - many functions that are "in sync with the development of the Web". Both traditional publishers and non-traditional content providers are interacting more with secondaries.

Auld takes a short-, medium- (six-month), and long-range view of the imperatives for secondary publishing, as follows.

Short-Term Imperatives:

link to the primary content;

have access to the historical file in the production mode;

develop a Web offering;

analyze marketing expenditures, and do more.

Medium-Term Imperatives:

make your Web offering a critical path;

develop cross-product customer files;

learn and understand Web "tools", e.g., graphic sound, instant messaging, e-mail;

take market research to the next level;

define content to include non-traditional offerings;

speed up the decision-making process.

Long-Term Imperatives:

develop market segmentation strategies;

evolve record and file content to be more dynamic;

analyze new roles. Are you going to become a filter or referee for primary material?;

establish brand;

look at the revenue mix - transactional commerce, advertising, and grants;

assess your position in the vertical integration to decide where to invest your resources.

Auld sees opportunity for secondaries in mounting "community support platforms" in step with the evolution of the Web.

"I too am very bullish on secondary publishers' function, but I am not necessarily convinced that in the year 2050 we will be the ones fulfilling that function", adds Bonnie Lawlor, President \& CEO of Chescot Publishing. Primary publishers and libraries have made some inroads into aggregation services today. She discusses factors shaping the future:

Current status of traditional secondary publishers - we must look at ourselves and what our core competencies are. We aggregate data and make it easier to use, but the growth in citation coverage is diminishing. Why? 
The evolution of higher education, and how this evolution will impact secondary information products. Distance education is on the rise. How does that change the information access behavior of a customer in the future?

User behavior. Everything is Web-oriented. Today's users are computer literate, have a need for immediate access, they seek answers, and prefer electronic sources (even if the information is inferior).

Definition of a publication - journal v. article. Who is going to create publications - author v. university v. publisher? How and who will index it?

The changing landscape of copyright law. There is a continuing tension between fair use and database protection. Will pending legislation bypass contract law?

Competition - from primary publishers, subscription agents, libraries, and universities. How will the Web search engines improve? Or will it be something totally different?

Lawlor urges a "sense of urgency" in responding to these factors.

"The core values of secondary publishing are at the heart of current and future information dissemination," says Linda Sacks, Senior Vice President, Marketing \& Sales, at BIOSIS. The major contributions of secondary publishing are aggregation, selection, integration, access points for retrieval, distribution, and archiving - essentially organizing the content. To Sacks, these core competencies represent what is currently lacking in today's Web environment. The synergies between Web publishing's inadequacies and the expertise that secondary publishers historically have in organizing content provide undisputed opportunities. 